Rubl. RIMS, Kyoto Univ.

12 Suppl. (1977), 247-256.

\title{
Local Cohomology of Analytic Spaces
}

\author{
by \\ Zoghman MEBKHOUT*
}

The purpose of this paper is to show that the local cohomology of a complex analytic space embedded in a complex manifold is a holonomic system of linear differential equations of infinite order and its holomorphic solution sheaves are a resolution of the constant sheaf $\boldsymbol{C}$ in this space which provides the Poincare lemma. The proof relies on the theories of the $b$-function and holonomic systems due to M. Kashiwara ([2] and [3]) and A. Grothendieck's theorem on the De Rham cohomology of an algebraic variety ([1]). I am very much indebted to M. Kashiwara from whose papers I learned so much.

\section{Notations}

We use the following notations:

$\left(X, \mathcal{O}_{X}\right) \quad$ : complex smooth manifold.

$Y \quad$ : reduce analytic subspace of $X$.

$I \quad:$ coherent ideal sheaf defining $Y$.

$\mathscr{D}_{X}{ }^{\infty}=\mathscr{D}^{\infty}$ : sheaf of differential operators on $X$.

$\mathscr{D}_{X}=\mathscr{D} \quad$ : sheaf of differential operators of finite order.

$D(\mathcal{A}) \quad$ : derived category of the category of $\mathcal{A}$-modules if $\mathcal{A}$ is any sheaf of rings.

A complex means a bounded complex. The sheaf $\mathscr{D}$ is coherent and the sheaf $\mathscr{D}^{\infty}$ is flat over $\mathscr{D}$.

\section{$\S$ 1. Main Theorems}

The local cohomology $\mathbb{R} \Gamma_{Y}\left(\mathscr{D}_{X}\right)$ of $Y$ is an object of $D\left(\mathscr{D}^{\infty}\right)$ because any injective $\mathscr{D}^{\infty}$-module is flabby. The algebraic local cohomology of $Y$ is the object of $D(\mathscr{D})$ defined instrinsically by

$$
\boldsymbol{R} \Gamma_{[X]}\left(\Theta_{X}=\mathbb{R} \lim _{\vec{k}} \operatorname{dom}_{\mathscr{D}_{X}}\left(\Theta_{X} / \mathcal{g}^{k} ; \mathcal{O}_{X}\right) .\right.
$$

Received July 14, 1976.

* Département de Mathématiques, U.E.R. Sciences Fondamentales et appliquées, 45045Orleans Cedex, France. 
Theorem 1. 1 i) The local algebraic cohomology is a complex with D-holonomic cohomology. ii) We have the canonical morphism in $D\left(\mathbb{C}_{X}\right)$

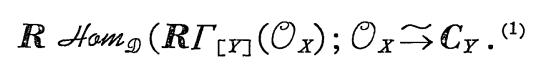

The natural morphism $\mathbb{R} \Gamma_{[Y]}\left(\Theta_{X}\right) \rightarrow \mathbb{R} \Gamma_{Y}\left(\Theta_{X}\right)$ induces a morphism in $D\left(\mathscr{D}^{\circ)}\right)$

$$
\mathscr{D}^{\infty} \bigotimes_{\mathscr{D}}^{L} \mathbb{R} \Gamma_{[Y]}\left(\Theta_{X}\right)=\mathscr{D}^{\infty} \bigotimes_{\mathscr{D}} \mathbb{R} \Gamma_{[Y]}(\Theta) \rightarrow \mathbb{R} \Gamma_{Y}\left(\Theta_{X}\right)
$$

Theorem 1.2 The morphism (*) is an isomorphism in $D\left(\mathscr{D}^{\infty}\right)$ and the local cohomology sheaves of $Y$ are $\mathscr{D}^{\infty}$-holonomic and admissible modules.

The theorem (1.1) is the Poincaré lemma because it gives a resolution of the constant sheaf $\mathbb{C}_{Y}$ in terms of analytic structure of $Y$. The flatness of $\mathscr{D}^{\infty}$ over $\mathscr{D}$ gives the following formula for every $p$ :

$$
\mathscr{H}_{Y}^{p}\left(\Theta_{X}\right)=\mathscr{D}^{\infty} \underset{\mathscr{D}}{\otimes} \underset{\vec{k}}{\lim _{x}} \mathcal{E}_{x} t^{p} \mathcal{C}_{X}\left(\mathcal{O}_{X} / \mathcal{I}^{k} ; \Theta_{X}\right)
$$

We get an expression of the local cohomology of a space in terms of the extension sheaves in analytic geometry which is useful in applications, for example in the proof of the theorem $B$. We have also the resolution

$$
\text { R } \operatorname{Hom}_{\mathscr{D}_{\infty}}\left(\mathbb{R} \Gamma_{Y}\left(\mathcal{O}_{X}\right) ; \mathcal{O}_{X} \underset{\rightarrow}{\rightarrow} C_{Y}\right. \text {. }
$$

We give the sketch of the proofs.

\section{$\S$ 2. The Algebraic Local Cohomology}

To be coherent with the notations [S.K.K.] we denote by $\mathfrak{B}_{Y \mid X}^{*}$ the cohomology of $\boldsymbol{R} \Gamma_{[Y]}\left(\Theta_{X}\right)$ [codim $\left.Y\right]$. To show that $\mathfrak{B}_{Y \mid X}^{*}$ are $\mathscr{D}$-holonomic we construct or canonical complex $L_{[Y]}\left(\Theta_{X}\right)$ on $X$ which has the same cohomology as $\mathbb{R} \Gamma_{[Y]}\left(\mathcal{O}_{X}\right)$. This complex reduces to the dualizing complex $L \cdot\left(\mathcal{O}_{X}\right)$ of J. P. Ramis and G. Ruget [8] if $Y=X$. We first suppose that $\operatorname{codim}(Y)=1$. In this case

(1) I was told by J. P. Ramis that he gets this formula with B. Malgrange by using cristalline cohomology. 


$$
L_{[Y]}\left(\mathcal{O}_{X}\right)=\boldsymbol{R} \Gamma_{[Y]}\left(\mathcal{O}_{X}\right) \underset{\vec{k}}{\underset{\lim }{\lim }} \mathcal{E}_{\mathcal{O}_{X}}^{1}\left(\mathcal{O}_{X} / \mathcal{I}^{k} ; \mathcal{O}_{X}\right)[-1]=\mathfrak{B}_{Y \mid X}^{1}[-1]
$$

To see that $\mathfrak{B}_{Y \mid X}^{1}$ is $\mathscr{D}$-holonomic system we can suppose that $Y=f^{-1}(0)$ where $f \in \Gamma\left(X, \mathcal{O}_{X}\right)$ and

$$
\mathfrak{B}_{Y \mid X}^{1} \underset{\rightarrow}{\rightarrow} \mathcal{O}_{X}\left[f^{-1}\right] / \mathcal{O}_{X}
$$

The singular supports $S S\left(\mathcal{O}_{X}\left[f^{-1}\right]\right)$ and $S S\left(\mathfrak{B}_{Y \mid X}^{1}\right)$ are the same because $S S\left(\Theta_{x}\right)$ is empty and it is enough to show that $\mathcal{O}_{x}\left[f^{-1}\right]$ is $\mathscr{D}$-holonomic. But it is just a consequence of the fundamental theorem of M. Kashiwara ([3]) which says that the $\mathscr{D}$-module $\Omega=\mathscr{D}[s] f^{s}$ is a coherent purely $(n-1)$-dimensional $\mathscr{D}$-module if $n=\operatorname{dim} X$.

Indeed, this theorem proves the the existence of the $b$-function of $f$ and this $b$-function gives

$$
\mathcal{O}_{x}\left[f^{-1}\right]=\mathscr{D} \cdot f^{-N}
$$

for a natural number $N$ large enough. We have the exact sequence

$$
0 \rightarrow(s+N) \mathscr{D}[s] f^{s} \rightarrow \mathscr{D}[s] f^{s} \rightarrow \mathscr{D} . f^{-N} \rightarrow 0 \text {. }
$$

This sequence shows that $\mathcal{O}_{x}\left[f^{-1}\right]$ is a coherent $\mathscr{D}$-module and a classical fact in dimension and multiplicity implies that $\operatorname{dim} S S\left(\Theta_{x}\left[f^{-1}\right]\right)=n-1$ which means that $\mathcal{O}_{X}\left[f^{-1}\right]$ is $\mathscr{D}$-holonomic. Let us define $L_{[Y]}\left(\Theta_{X}\right)$ when $\operatorname{codim}(Y) \geq 2$. Remember that for any regular noetherian scheme $\left(\widetilde{X}, \mathcal{O}_{\widetilde{X}}\right)$ over $\mathbb{C}$ the cousin complex,

$$
L \cdot\left(\mathcal{O}_{\widetilde{X}}\right)=\mathcal{H}_{Z \cdot \mid Z_{+1}}\left(\mathcal{\Theta}_{\widetilde{X}}\right)
$$

is an injective hence a flabby resolution of $\mathcal{O}_{\widetilde{X}}$. For a compact $K \subset X$ we denote by $\left(\widetilde{X}(K), \mathcal{O}_{\widetilde{X}(K)}\right)$ the affine scheme defined by $\mathcal{O}(K)$ and by $\widetilde{Y}(K)$ the subscheme defined by the ideal $I(K)$ of the vanishing functions on $Y$ in a neighborhood of $K$. For a point $x \in X$ the fiber of $L_{[Y]}\left(\mathcal{O}_{X}\right)$ at $x$ will be $\Gamma_{\widetilde{Y}(x)}\left(L \cdot\left(\mathcal{O}_{\widetilde{X}(x)}\right)\right)$ which is just the local cohomology of $\widetilde{Y}(x)$ in the local scheme $\widetilde{X}(x)$. To glue the different fibers we take a compact polycylinder $K$, the ring $O(K)$ is noetherian and the cousin complex $L \cdot\left(\mathcal{O}_{\widetilde{X}(K)}\right)$ is a flabby resolution of $\mathcal{O}_{\widetilde{X}(K)}$. If $x \in \stackrel{\circ}{K}$, the morphism $\mathcal{O}(K) \rightarrow \mathcal{O}_{x}$ gives a morphism

$$
\Gamma_{\widetilde{X}(K)}\left(L \cdot\left(\mathcal{O}_{\widetilde{X}(K)}\right)\right) \rightarrow \Gamma_{\widetilde{Y}(x)}\left(L \cdot\left(\mathcal{O}_{\widetilde{X}(x)}\right)\right) .
$$

Lemma 2.1 When Kruns over the neighborhoods of $x$, the morphism 
$\lim \Gamma_{\widetilde{Y}(K)}(L \cdot(\varrho \widetilde{X}(K))) \rightarrow \Gamma_{\widetilde{Y}(x)}\left(L \cdot\left(\mathcal{O}_{\widetilde{X}(x)}\right)\right)$ is an isomorphism. $\overrightarrow{K_{\ni} x}$

This lemma glues together the fibers and and the complex $L_{\left[{ }^{X}\right]}\left(\mathcal{O}_{X}\right)$ will have the same cohomology as $\boldsymbol{R} \Gamma_{[Y]}\left(\mathcal{O}_{X}\right)$ because of the expression of the local cohomology of a closed space in a noetherian scheme in terms of the extensions. Now to prove that $\mathfrak{B}_{Y \mid X}^{*}$ are coherent $\mathscr{D}$-modules it is enough to prove that for any small polycylinder $K \Gamma\left(K, \mathfrak{B}_{Y \mid X}^{*}\right)$ are $\mathscr{D}(K)$-module of finite type and that the morphism

$$
\Gamma\left(K, \mathfrak{B}_{Y \mid X}^{*}\right) \bigotimes_{\mathcal{O}(K)} \mathcal{O}_{x} \rightarrow \mathfrak{B}_{Y \mid X, x}^{*}
$$

is an isomorphism for every $x \in \stackrel{\circ}{K}$ (see [6]). If $f_{1}, \cdots, f_{q}(q \geqq 2)$ are functions of $\mathcal{O}(K)$ defining $Y$ in a neighborhood of $K$ we can compute $\Gamma\left(K, \mathfrak{B}_{Y \mid X}^{*}\right)$ as the Čech cohomology of the Zariski-Čech covering $\mathfrak{U}=\bigcup_{i} U_{i}$ of $\widetilde{X}(K) \backslash \widetilde{Y}(K)$ where $U_{i}=\widetilde{X}(K) \backslash V\left(f_{i}\right)$. The Čech complex $C \cdot\left(\mathfrak{U}, \mathcal{O}_{\widetilde{X}(K)}^{i}\right)$ is a complex of $\mathscr{D}(K)$-module of finite type in vertue of the codimension one so $\Gamma\left(K, \mathfrak{B}_{Y \mid X}^{*}\right)$ are of finite type. The module $\mathcal{O}_{x}$ is flat over $\mathcal{O}(K)$ if $x \in \stackrel{\circ}{K}$ so the tensor product with $\mathcal{O}_{x}$ over $\mathcal{O}(K)$ commutes with the cohomology. But we have in vertue of the one codimensionality the following isomorphism,

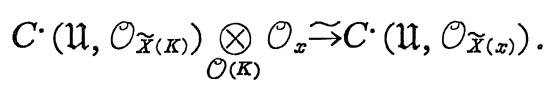

Taking the cohomology in the both hand side we have the isomorphism

$$
\Gamma\left(K, \mathfrak{B}_{Y \mid X}^{*}\right) \underset{\mathcal{O}(K)}{\bigotimes} \mathcal{O}_{x} \underset{\rightarrow}{\rightarrow} \mathfrak{B}_{Y \mid X, x}^{*},
$$

and the $\mathscr{D}$-modules $\mathfrak{B}_{Y \mid X}^{*}$ are coherent. To see that these modules are holonomic we just notice that the object of the complex $\Gamma_{\widetilde{Y}(x)}\left(L \cdot\left(\mathcal{O}_{\widetilde{X}(x)}\right)\right)$ have dimensions $n-1$ as $\mathscr{D}_{x}$-modules if we compute it by the ČechZariski cohomology.

\section{$\S 3$. The De Rham Complex of $R \Gamma_{[Y]}\left(\mathscr{D}_{X}\right)$.}

We denote by $T$ the tangent vector bundle of $X$ and by $\Omega^{\circ}{ }_{X}=\Omega$. the De Rham complex of $X$. The first Spencer sequence $\mathscr{D} \otimes_{\mathcal{O}_{X}} \Lambda^{\cdot}(T)$ is a projective resolution of $\mathcal{O}_{X}$ in the category of $\mathscr{D}$-modules. For any complex $\mathscr{M} \cdot$ of $\mathscr{D}$-modules we have 


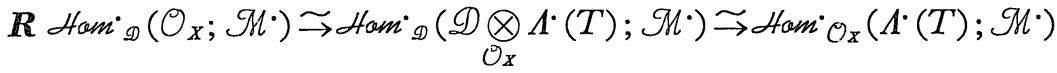

$$
\begin{aligned}
& =\Omega^{\cdot}{ }_{\partial_{x}} \otimes \mathcal{M} \cdot \text {. }
\end{aligned}
$$

These isomorphisms hold in $D\left(\mathbb{C}_{X}\right)$. The complex $\boldsymbol{R}$ Hom $_{\mathscr{D}^{\circ}}\left(\mathcal{O}_{X} ; \mathscr{M}^{*}\right)$ is called the De Rham complex of $\mathcal{M}^{\cdot}$ and is denoted by $D R\left(\mathcal{H}^{*}\right)$ By the Poincaré lemma we have $\boldsymbol{R} \operatorname{Hom}_{\mathscr{D}}\left(\mathcal{O}_{X} ; \mathcal{O}_{X}\right) \underset{\rightarrow}{\rightarrow} \stackrel{\sim}{\rightarrow} \mathbb{C}_{X}$ in $D\left(\mathbb{C}_{X}\right)$. The naturel injection $\boldsymbol{R} \Gamma_{[\mathrm{Y}]}\left(\Theta_{X}\right) \rightarrow \boldsymbol{R} \Gamma_{Y}\left(\Theta_{X}\right)$ gives a morphism

$$
\begin{aligned}
& \mathbb{R} \operatorname{Ham}_{\mathfrak{D}}\left(\mathcal{O}_{X} ; \boldsymbol{R} \Gamma_{[Y]}\left(\Theta_{X}\right)\right) \rightarrow \mathbb{R} \operatorname{Ham}_{\mathscr{D}}\left(\mathcal{O}_{X} ; \mathbb{R} \Gamma_{Y}\left(\mathcal{O}_{X}\right)\right)
\end{aligned}
$$

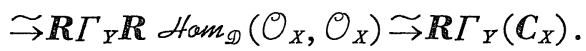

Theorem 3.1 The composed morphism $\mathbb{R}$ Ham $_{\mathscr{D}}\left(\mathcal{O}_{X} ; \mathbb{R} \Gamma_{[Y]}\left(\mathcal{O}_{X}\right)\right)$ $\rightarrow \boldsymbol{R} \Gamma_{Y}\left(\boldsymbol{C}_{X}\right)$ is an isomorphism in $D\left(\boldsymbol{C}_{X}\right)$.

The question is local. We can suppose that $\mathcal{G}=\left(f_{1}, \cdots, f_{q}\right)$. Let $\mathcal{I}_{1}=\left(f_{1}, \cdots, f_{q-1}\right), \mathcal{I}_{2}=\left(f_{q}\right)$ and $Y_{1}$ and $Y_{2}$ the spaces defined by $\mathcal{I}_{1}$ and $\mathcal{I}_{2}$. We have $Y=Y_{1} \cap Y_{2}$ and $Y_{1} \cup Y_{2}$ is defined by $\left(f_{1} f_{q}, \cdots, f_{q-1} f_{q}\right)$. We have a triangle

$$
\begin{gathered}
\boldsymbol{R} \Gamma_{\left[Y_{1} \cup Y_{2}\right]}\left(\mathcal{O}_{X}\right) \\
+1 \swarrow \\
\boldsymbol{R} \Gamma_{[Y]}\left(\mathcal{O}_{X}\right) \longrightarrow \mathbb{R} \Gamma_{\left[Y_{1}\right]}\left(\mathcal{O}_{X}\right) \oplus \boldsymbol{R} \Gamma_{\left[X_{2}\right]}\left(\Theta_{X}\right)
\end{gathered}
$$

To see that (1) is a triangle in $D(\mathscr{D})$, it is enough to see it on each fiber because of the nature of the Cousin complex. But if $x \in X$ the triangle

$$
\begin{aligned}
& +1 \stackrel{\boldsymbol{R} \Gamma_{\left[Y_{1} \cup Y_{2}\right]}\left(\mathcal{O}_{X}\right), x}{\nwarrow} \\
& \boldsymbol{R} \Gamma_{[Y]}\left(\mathcal{O}_{X}\right),{ }_{x} \longrightarrow \mathbb{R} \Gamma_{\left[Y_{1}\right]}\left(\mathcal{O}_{X}\right),{ }_{x} \oplus \boldsymbol{R} \Gamma_{\left[Y_{2}\right]}\left(\mathcal{O}_{X}\right),{ }_{x}
\end{aligned}
$$

is just the Mayer-Vietoris sequence of the subspaces $\widetilde{Y}_{1}(x)$ and $\widetilde{Y}_{2}(x)$ in the scheme $\widetilde{X}(x)$. We can also use Artin-Rees lemma and cofinality. The functor $\boldsymbol{R}$ Ham $_{\mathscr{D}}\left(\mathcal{O}_{X} ; *\right)$ from $D(\mathscr{D})$ to $D\left(\mathbb{C}_{X}\right)$ is a $\partial$-functor and transforms triangle (1) into the triangle (2)

$$
\begin{aligned}
& \quad D R\left(\boldsymbol{R} \Gamma_{\left[Y_{1} \cup Y_{2}\right]}\left(\mathcal{O}_{X}\right)\right) \\
& +1 \swarrow \quad \\
& D R\left(\boldsymbol{R} \Gamma_{[Y]}\left(\Theta_{X}\right)\right) \longrightarrow D R\left(\mathbb{R} \Gamma_{\left[Y_{1}\right]}\left(\Theta_{X}\right)\right) \oplus D R\left(\boldsymbol{R} \Gamma_{\left[Y_{2}\right]}\left(\Theta_{X}\right)\right) .
\end{aligned}
$$


The Mayer-Vietoris sequence of $Y_{1}$ and $Y_{2}$ in $X$ gives the triangle (3) (3)

$$
\begin{aligned}
& \mathbb{R} \Gamma_{Y_{1} \cup Y_{2}}\left(\mathbb{C}_{X}\right) \\
& +1 \swarrow \\
& \mathbb{R} \Gamma_{Y}\left(\mathbb{C}_{X}\right) \longrightarrow \mathbb{R} \Gamma_{Y_{1}}\left(\mathbb{C}_{X}\right) \oplus \mathbb{R} \Gamma_{Y_{2}}\left(\mathbb{C}_{X}\right)
\end{aligned}
$$

The morphism of the theorem 3.1 is a morphism of the triangle (2) to triangle (3). By induction on $q$ the proof of the theorem 3.1 is reduced to the case $\mathcal{G}=(f)$. If $Y=f^{-1}(0)$ let $U$ be $X \backslash Y$ and $j$ the injection of $U$ in $X$. In this case

$$
\boldsymbol{R} \Gamma_{[Y]}\left(\mathcal{O}_{X}\right)=\mathfrak{B}_{Y \mid X}^{1}[-1]=\mathcal{O}_{X}\left[f^{-1}\right] / \mathcal{O}_{X}[-1]
$$

We have the triangle in $D\left(\boldsymbol{C}_{X}\right)$

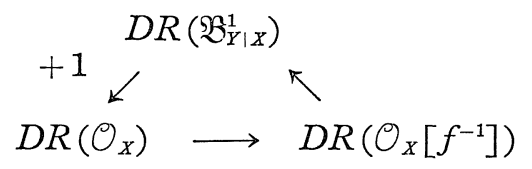

and the triangle in $D\left(\mathbb{C}_{X}\right)$

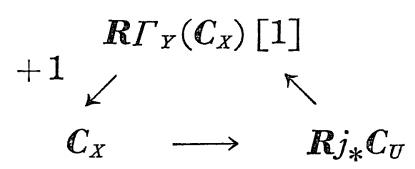

But the following composed morphism

$$
D R\left(\mathcal{O}_{X}\left[f^{-1}\right]\right) \rightarrow j_{*} \Omega^{\cdot}{ }_{U} \widetilde{\rightarrow} \mathbb{R} j_{*} \Omega^{\cdot}{ }_{U} \rightarrow \boldsymbol{R} j_{*} \boldsymbol{C}_{U}
$$

is an isomorphism by the Grothendieck's theorem [1]. Finally we get

$$
\boldsymbol{R} \operatorname{Hom}_{\mathscr{D}}\left(\mathcal{O}_{X} ; \boldsymbol{R} \Gamma_{[Y]}\left(\mathcal{O}_{X}\right)\right) \stackrel{R}{\rightarrow} \Gamma_{Y}\left(C_{X}\right)
$$

and the proof of theorem (3.1) is complete.

\section{§4. Verdier Duality}

Remember that a $\mathbb{C}$-analytic finitistic sheaf of $\mathbb{C}$ vector spaces ( $\mathbb{C}$ analytiquement constructible in French) is a sheaf $\mathscr{F}$ of finite $\mathbb{C}$-vector spaces such that there exists a stratification $\bigcup_{i} X_{i}$ of $X$ and the restriction $\left.F\right|_{X_{i}}$ on each stratum is locally constant. By M. Kashiwara [2] the complex $\mathbb{R} \operatorname{Ham}_{\mathscr{D}}\left(\mathscr{M}, \mathcal{O}_{X}\right)$ has finitistic cohomology if $\mathscr{M}$ is a $\mathscr{D}$-holonomic system. Because the category of finitistic sheaves is a stick subcategory 
of $\mathbb{C}$-vector spaces the complex $\mathbb{R}$ Hom $_{\mathscr{D}}\left(\mathcal{H} \cdot \mathcal{O}_{X}\right)$ has finitistic cohomology if $\mathscr{M} \cdot$ is a complex with $\mathscr{D}$-holonomic cohomology, We call a complex with finitistic cohomology a finitistic complex. So by the first part of theorem 1.1 the complex $\mathbb{R} \operatorname{Ham}_{\mathscr{D}}\left(\mathbb{R} \Gamma_{[Y]}\left(\mathcal{O}_{X}\right) ; \mathcal{O}_{X}\right)$ is finitistic. Using the topological duality of Verdier and devissage [12] we can see that $\boldsymbol{R} \operatorname{Hom}_{\boldsymbol{C}_{X}}\left(\mathscr{F}^{\cdot}, \boldsymbol{C}_{X}\right)$ is finitistic if $\mathscr{F}^{\cdot}$ is a finitistic complex and the natural morphism

$$
\mathscr{F} \cdot \rightarrow \mathbb{R} \operatorname{Ham}_{C_{X}}\left(\mathbb{R} \operatorname{Hom}_{C_{X}}\left(\mathscr{F} \cdot, \mathbb{C}_{X}\right) ; \mathbb{C}_{X}\right)
$$

is an isomorphism in $D\left(\mathbb{C}_{X}\right)$. The sheaf $\mathbb{C}_{Y}$ is finitistic and we have

$$
\mathbb{C}_{Y} \rightarrow \mathbb{R} \operatorname{Ham}_{C_{X}}\left(\mathbb{R} \operatorname{Ham}_{C_{X}}\left(\mathbb{C}_{Y} ; \mathbb{C}_{X}\right) ; \mathbb{C}_{X}\right) \text {. }
$$

We have also

$$
\begin{aligned}
\mathbb{R} \operatorname{Ham}_{\mathscr{D}} & \left(\mathbb{R} \Gamma_{[Y]}\left(O_{Y}\right) ; O_{Y}\right) \\
& \simeq \mathbb{R} \operatorname{Ham}_{\boldsymbol{C}_{X}}\left(\mathbb{R} \operatorname{Ham}_{\boldsymbol{C}_{X}}\left(\mathbb{R} \operatorname{Ham}_{\mathscr{D}}\left(\mathbb{R} \Gamma_{[Y]}\left(\Theta_{Y}\right) ; \Theta_{X}\right) ; \mathbb{C}_{X}\right) ; \mathbb{C}_{X}\right)
\end{aligned}
$$

To prove that $\mathbb{C}_{X} \widetilde{R} \mathbb{H}_{\mathbb{D}}\left(\mathbb{R} \Gamma_{[X]}\left(\Theta_{X}\right) ; \Theta_{X}\right)$ it suffices to prove that $\mathbb{R} \operatorname{Htam}_{\mathbb{C}_{X}}\left(\mathbb{C}_{Y} ; \mathbb{C}_{X}\right)=\mathbb{R} \Gamma_{Y}\left(\mathbb{C}_{X}\right) \underset{\rightarrow}{\mathbb{R}} \operatorname{Ham}_{C_{X}}\left(\mathbb{R} \operatorname{Ham}_{\mathscr{D}}\left(\mathbb{R} \Gamma_{[Y]}\left(\Theta_{X}\right) ; \Theta_{X}\right) ; \mathbb{C}_{X}\right)$. By theorem 3.1 we have $\mathbb{R} \Gamma_{Y}\left(\mathbb{C}_{X}\right)=\mathbb{R} \operatorname{Ham}_{\mathscr{D}}\left(\Theta_{X} ; \mathbb{R} \Gamma_{[Y]}\left(\mathcal{O}_{X}\right)\right)$ and we must prove that

$$
\mathbb{R} \operatorname{Ham}_{\mathscr{D}}\left(\mathcal{O}_{X} ; \mathbb{R} \Gamma_{[Y]}\left(\Theta_{X}\right)\right) \underset{\rightarrow}{\mathbb{R}} \operatorname{Ham}_{C_{X}}\left(\mathbb{R} H_{a m}\left(\mathbb{R} \Gamma_{[Y]}\left(\Theta_{X}\right) ; \Theta_{X}\right) ; \mathbb{C}_{X}\right) \text {. }
$$

This can be done by the following theorem which completes the proof of the theorem $(1,1)$ :

Theorem 4. 1 Let $\mathscr{M} \cdot$ be a complex with D-holonomic cohomology then we have a canonical isomorphism in $D\left(\mathbb{C}_{X}\right)$

$$
\mathbb{R} \operatorname{Ham}_{\mathscr{D}}\left(\mathcal{O}_{X} ; \mathscr{H} \cdot\right) \stackrel{\mathbb{R}}{\rightarrow} \operatorname{Ham}_{C_{X}}\left(\mathbb{R} \operatorname{Ham}_{\mathscr{D}}\left(\mathscr{M} ; \Theta_{X}\right) ; \mathbb{C}_{X}\right) \text {. }
$$

\section{§5. T.V.S. Homological Algebra}

To prove theorem 4.1 and theorem 1.2 we need to define the functor $\mathbb{R}$ Homtop $^{\circ} \boldsymbol{C}_{X}\left(\mathscr{F}^{\prime} ; \mathscr{L}^{*}\right)$ if $\mathscr{F}^{\cdot}$ and $\mathscr{L} \cdot$ are complex of $\mathscr{D}_{X}$-module locally free with differential operators of finite order. Roughly speeking it is the "derived" functor of $\operatorname{Hantap}_{\boldsymbol{C}_{X}}(\mathscr{F} ; \mathcal{G})$ which represents the continuous homomorphisms of Fréchet-nuclear sheaves. This category 
is not abelian. J. P. Ramis had noticed [10] and [11] that $\boldsymbol{C}_{X}$ is just the Libermann complex $\boldsymbol{B}^{\cdot}[5]$ and using the graded ring $\boldsymbol{B} \cdot$ he could define $\boldsymbol{R}$ Hamtop $_{\boldsymbol{C}_{X}}(\mathscr{F} ; \mathcal{L})$. We do not give here the precise definition but we recall the formula

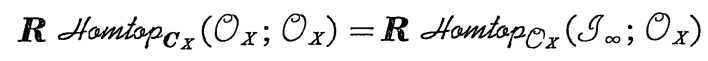

where $\mathcal{G}_{\infty}$ is the sheaf of infinite jets; see [9]. We list the properties of this functor in the following theorem:

Theorem 5. 1 We have the following isomorphisms;

a) $\boldsymbol{R}$ Homtsp $_{\boldsymbol{C}_{X}}\left(\Theta_{X} ; \Theta_{X}\right) \underset{\rightarrow}{\rightarrow} D^{\infty}$

b) $\boldsymbol{R}$ Homtop $_{\boldsymbol{C}_{X}}\left(\mathcal{O}_{X} ; \Omega^{\cdot}\right) \stackrel{\rightarrow}{\rightarrow} \Omega^{n}[-n]$

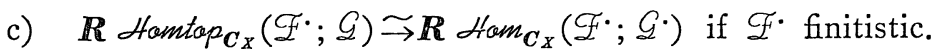

We can now prove theorem 4.1. The natural morphism

$$
\operatorname{Ham}_{C_{X}}(\mathscr{I} ; \mathscr{L}) \underset{\mathscr{D}}{\otimes} \mathscr{H} \cdot \rightarrow \operatorname{Ham}_{\boldsymbol{C}_{X}}\left(\operatorname{Ham}_{\mathscr{D}}(\mathscr{H} \cdot ; \mathscr{F}) ; \mathcal{L}\right),
$$

where $\mathscr{F}^{\cdot}$ and $\mathscr{M} \cdot$ are left $\mathscr{D}$-modules, gives rise to the morphism of functors

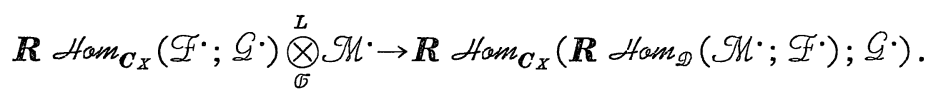

Notice that the structure of right $\mathscr{D}$-module of $\operatorname{Ham}_{C_{X}}\left(\mathscr{F} \cdot ; \mathscr{Q}^{\cdot}\right)$ comes from the structure of left $\mathscr{D}$-module $\mathscr{F}$. This morphism of functors is an isomorphism if $\mathscr{N} \cdot$ has $\mathscr{D}$-coherent cohomology by the way out "left" functor lemma. We have a natural morphism in $D\left(\mathbb{C}_{X}\right)$

$$
\boldsymbol{R} \text { Homtop }_{\boldsymbol{C}_{X}}\left(\mathrm{O}_{X} ; \Omega^{*}\right) \rightarrow \boldsymbol{R} \operatorname{Ham}_{C_{X}}\left(\mathcal{O}_{X} ; \Omega^{\cdot}\right)
$$

which give a morphism by composition with the last one (*) $\quad \boldsymbol{R}$ Hamtap $_{\boldsymbol{C}_{X}}\left(\mathcal{O}_{X} ; \Omega^{\cdot}\right) \bigotimes_{\widetilde{L}}^{L} \mathcal{M} \cdot \rightarrow \boldsymbol{R} \operatorname{Ham}_{C_{X}}\left(\boldsymbol{R} \operatorname{Ham}_{\mathscr{D}}\left(\mathcal{M} \cdot ; \mathcal{O}_{X}\right) ; \Omega \cdot\right)$.

Theorem 5.2 Let $\mathscr{M} \cdot$ a complex with D-holonomic cohomology then (*) is an isomorphism in $D\left(\mathbb{C}_{X}\right)$.

The question is local. We can suppose that $\mathscr{M}^{\cdot}$ is a single holono- 
mic $\mathscr{D}$-module admitting a free resolution. In this case $\boldsymbol{R} \operatorname{Ham}_{\mathscr{D}}\left(\mathscr{M} ; \mathcal{O}_{X}\right)$ is a complex of free $\mathcal{O}_{X}$-modules with differential being differential operators of finite order and $\boldsymbol{R} \operatorname{Hamtap}_{C_{X}}\left(\boldsymbol{R} \operatorname{Ham}_{\mathscr{D}}\left(\mathcal{M} ; \mathcal{O}_{X}\right) ; \Omega^{*}\right)$ has a meaning. The morphism (*) transit via the morphism $\mathbb{R}$ Hamtop $_{\boldsymbol{C}_{X}}$ $\times\left(\mathcal{O}_{X} ; \Omega^{\cdot}\right) \bigotimes_{\mathscr{D}}^{L} \mathscr{M} \rightarrow \boldsymbol{R}$ Homtap $_{\boldsymbol{C}_{X}}\left(\boldsymbol{R}\right.$ Ham $\left.\left(\mathscr{M} ; \mathcal{O}_{X}\right) ; \Omega^{\cdot}\right)$. The last morphism is an isomorphism by the technique of the way out "left" functor lemma. The theorem 5.2 is a consequence of the property c) of theorem (5.1) because $\boldsymbol{R} \operatorname{Ham}_{\mathscr{D}}\left(\mathscr{M} ; \mathcal{O}_{X}\right)$ is finitistic. In $D\left(\mathbb{C}_{X}\right)$ we have $\Omega: \widetilde{\rightarrow} \mathbb{C}_{X}$ and

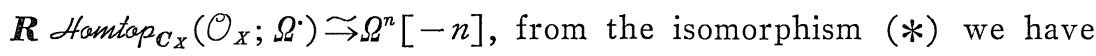

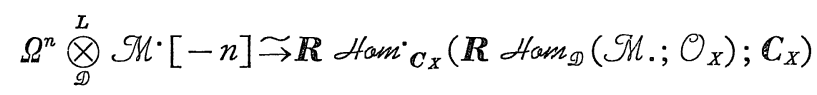

and the theorem 4.1 follows if we notice that

$$
\Omega^{n} \stackrel{L}{\otimes} \mathscr{D} \cdot[-n] \widetilde{\rightarrow} D R(\mathscr{M} \cdot)=\mathbb{R} \operatorname{Han}_{\mathscr{D}}\left(\mathcal{O}_{X} ; \mathscr{M}^{\cdot}\right)
$$

\section{§ 6. Local Cohomology of $\mathbb{Y}$.}

The natural morphism of functors in the argument $\mathcal{M}^{\text {. }}$

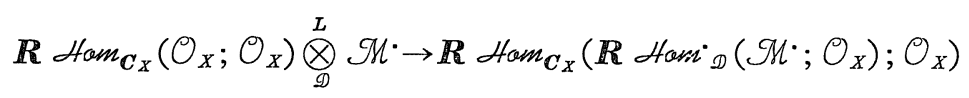

is an isomorphism if $\mathscr{M} \cdot$ has $\mathscr{D}$-coherent cohomology by the way out left functor. The natural morphism

$$
\boldsymbol{R} \text { Homtop }_{\boldsymbol{C}_{X}}\left(\mathcal{O}_{X} ; \mathcal{O}_{X}\right) \rightarrow \mathbb{R} \operatorname{Ham}_{C_{X}}\left(\mathcal{O}_{X} ; \mathcal{O}_{X}\right)
$$

gives the morphism

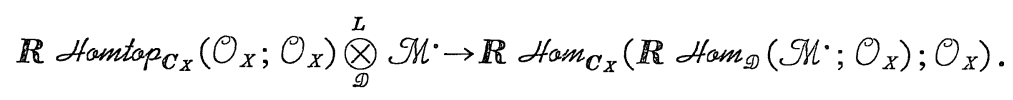

Proposition 6.1 The morphism (*) is an isomorphism in $D\left(\mathbb{C}_{X}\right)$ if $\mathscr{M} \cdot$ has $\mathscr{D}$-holonomic cohomology.

The question is local. We can suppose that $\mathscr{M}^{\cdot}$ is a single $\mathscr{D}$ holonomic system admitting a free resolution. We finish the proof in the same way as in the last section We apply this situation to $\mathscr{M}$. $=\mathbb{R} \Gamma_{\left[{ }_{[Y]}\right.}\left(\mathcal{O}_{X}\right)$. We have 


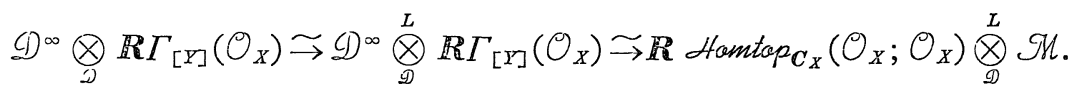

$$
\begin{aligned}
& \underset{\rightarrow}{R} \operatorname{Ham}_{C_{X}}\left(\mathbb{R} \operatorname{Hom}_{\mathscr{D}}\left(\mathbb{R} \Gamma_{[Y]}\left(\Theta_{X}\right) ; \Theta_{X}\right) ; \Theta_{X}\right)
\end{aligned}
$$

and by theorem $1.1 \mathbb{R} \operatorname{Ham}_{\mathscr{D}}\left(\mathbb{R} \Gamma_{[Y]}\left(\mathcal{O}_{X}\right) ; \mathcal{O}_{X}\right) \underset{\rightarrow}{\rightarrow} \mathbb{C}_{Y}$. So

$$
\mathscr{D}^{\infty} \otimes_{\mathscr{D}} \mathbb{R} \Gamma_{[Y]}\left(\Theta_{X}\right) \stackrel{\mathbb{R}}{\rightarrow} \operatorname{Ham}_{\boldsymbol{C}_{X}}\left(\boldsymbol{C}_{Y} ; \Theta_{X}\right)=\mathbb{R} \Gamma_{Y}\left(\Theta_{X}\right) \text {. }
$$

The proof of theorem 1.2 is over. The details will appear elsewhere.

\section{References}

(S.K.K.) Sato, M., Kawai, T. and Kashiwara, M., Microfunctions and pseudo-differential equations, Lecture note in Math., 287, Springer, Heidelberg-New York 265-529, 1973.

[1] Grothendieck, A., On the De Rham Cohomology of algebraic varieties, Publ. Math. I.H.E.S; 29 (1966), 95-103.

[2] Kashiwara, M., On the maximally overdetermined systems of linear differential equation I*. Publ. R.I.M.S. Kyoto Univ. 10 (1975), 563-579.

[3] Kashiwara, M., Lettre à Malgrange Janvier (1975). On the rationality of the roots of $b$-functions.

[4] Libermann, D. and Herrera, M., Duatity and the De Rham Cohomology of infinitesimal neighborhoods, Invent. Math., 13 (1971), 97-326.

[5] Libermann, D., Generalizations of the De Rham Complex with applications to duality theory and the cohomology of singular varieties, Proc. conf. of Complex Analysis, Rice, 1972.

[6] Malgrange, B., Pseudo-differentiels operateurs-Seminaire Grenoble (1976).

[7] Malgrange, B. et Ramis, J. P., (to appear)

[8] Ramis J. P. et Ruget. G., Complex dualisant en geometrie analytique, Publ. I.H.E.S., 38, 77-91 (1971).

[9] Ramis, J. P. et Ruget, G., Dualité et résidu, Invent. Math., 26 Fasc 2, (1974), 89131.

[10] Ramis, J. P., Lettre à Malgrange Janvier 1976)

[11] Ramis, J. P., Lettre à Verdier (Février 1976)

[12] Verdier, J. L., Classe d'homologie d'un cycle séminaire Douady-Verdier E.N.S. (1975). 